\title{
Modes of Bifurcations, Chaos, and Undamped Oscillations in a Buck Converter with Feedback and Their Synchronizing
}

\author{
Yuval Beck ${ }^{1}$, Yefim Berkovich ${ }^{2 *}$ \\ ${ }^{1}$ School of Electrical Engineering, Tel Aviv University (TAU), P.O. Box 39040, Tel Aviv 69978, Israel. \\ 2 Department of Electrical Engineering, Holon Institute of Technology, 52 Golomb st., Holon, 58102, Israel. \\ *Corresponding author. Tel.: +97235026632; email: Yefim.Berkovich@gmail.com. \\ Manuscript submitted April 09, 2019; accepted July 30, 2019. \\ doi: 10.17706/ijcee.2019.11.4.164-174
}

\begin{abstract}
This paper considers special functioning modes of a buck converter under closed loop feedback, which, depending on the type of a regulator, are characterized by the appearance of bifurcations which are transformed into the chaotic mode, or by undamped oscillations. The paper presents a simple and compact mathematical model. The model is implemented in the Matlab program, which makes it possible to carry out various calculations, including the cyclic ones, followed by graphic and numerical data processing. For normalizing the performance of buck converters, a new method of synchronizing the occurring modes proposed, and its efficiency is tested for various regulators in a feedback system. The accuracy of the proposed mathematical model, and of the adequacy of the obtained results, are tested by the Matlab-Simulink program.
\end{abstract}

Key words: Buck converter, control, bifurcation, chaos.

\section{Introduction}

Physical models based on electric circuits are convenient for studying chaotic modes of varius origins, which are studied by new scientific disciplines such as nonlinear dynamics, synergetics, and generalized information theory [1]-[6].

An example of such a simple model is the a boost converter, which transforms a direct voltage to a higher voltage value, and which is operating in a Current Mode Control (CMC) mode. There are various works which are devoted to the investigation of chaotic modes in boost converters, [7]-[9], as well as the works of C. K. Tse [10]-[12].

Besides a boost converter that operates in CMC mode, a convenient and a very simple model is the common step-down buck converter with a closed loop system of automatic control and a proportional regulator. The work in [13] considers this model very meticulously and gives detailed analytical and numerical calculations of processes in a buck converter in a closed loop feedback under some changes in the input voltage. The work describes various versions of the sawtooth supporting voltage, as well as some methods of synchronization of chaotic modes. One of them is based on [14], where it is proposed to introduce small increments to some values of the system, another method shown in [15], noted as Time-Delayed auto synchronization (TDAS), introduces a control signal formed with a difference between the current state of the system and the state of the system delayed by one period of the unstable periodic 
orbit (UPO). Two methods of chaos control are based on feedback combined with an external oscillator or time-shifted feedback suggested in [16].

In [17] the investigation of chaotic regimes in a buck converter was carried out in three different ways and the results are compared. There, three independent cases are compared: the mathematical model simulated in Matlab, the circuit built from components that exist in reality and simulation in PSpice as well as a laboratory experiment. In [18] bifurcation and chaos in high-frequency peak current mode buck converter working in continuous conduction mode (CCM) are studied : a two-dimensional discrete mapping model is established, reference current at the period-doubling point and the border of inductor current are derived and the bifurcation diagrams are drawn with the aid of MATLAB. Furthermore, circuit simulations are executed with PSIM. A new computationally-based methodology to obtain chaos regulators for PWM buck power converters is proposed in [19]. The technique there is based on a feed-forward control, where the offset of the T-periodic sawtooth signal is modified continuously, and the sawtooth is redefined as a function of the output and reference voltages.

It should be noted that while many papers provide a very detailed descriptions of the electric modes, there is much less attention to paid to the models themselves and to the means of calculations based on these models. Thus, these models should be considered and further investigated including new algorithms for data processing. The methods for removing chaotic modes are based on creating special regulators that perform various calculation procedures based on mathematical interpretations of these processes without touching the physical essence of their course.

This paper presents a simple mathematical model, which makes it possible to generate and investigate general laws of the development of chaotic processes. The model provides both means for obtaining the needed modes, as well as their mathematical processing.

The paper is organized as follows: Section 2 describes the model for the case of a proportional regulator in the feedback circuit, and in Section 4, for an integral, or a proportional-integral regulators. Sections 3, 5 and 6 propose new simple methods of synchronizing (preventing) chaotic modes, which are based on the action on the power scheme of the converter, whose efficiency has been proved.

\section{The Mathematical Model of a Buck Converter with a Closed Feedback and a Proportional Regulator (P-Regulator)}

In Fig. 1 a diagram of a buck converter with a closed loop control system is shown. Its operation is well-known and demands no additional explanations. The dynamic behavior of the buck converter is given by a system of differential equations with respect to the inductor's current $i_{o}$ and the output voltage, $v_{o}$ as follows:

$$
\begin{aligned}
& L_{o} d i_{o} / d t+v_{o}=d \cdot V_{i n} \\
& C_{o} d v_{o} / d t+v_{o} / R_{o}=i_{o}
\end{aligned}
$$

where $d$ is a binary switching function, which equals " 1 ", when the transistor $S$ is conducting, and " 0 ",

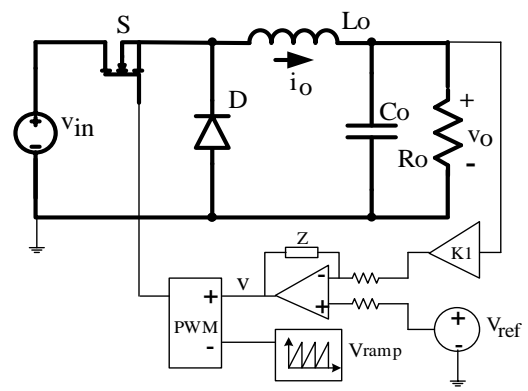

Fig. 1. A buck converter with a closed loop control. 
when it is disconnected. When $d=" 0$ " the diode $D$ is conducting, and the voltage at the input of the circuit $L_{o}-R_{o}-C_{o}$ equals zero.

Let us replace the differentials of the current and the voltage with their finite increments $\Delta i_{o}=i_{o}(\Delta t(k+1))-i_{o}(\Delta t \cdot k)$ and $\Delta v_{o}=v_{o}(\Delta t(k+1))-v_{o}(\Delta t \cdot k)$ for sufficiently short time intervals $\Delta t$ on each $\mathrm{k}^{\text {th }}$ segment of the calculation and modify the form of writing of the system of equations (1) such that,

$$
\begin{aligned}
& i_{o}(\Delta t(k+1))=\left(d \cdot V_{\text {in }} / L_{o}-v_{o}(\Delta t \cdot k) / L_{\text {in }}\right) \cdot \Delta t+i_{o}(\Delta t \cdot k) \\
& v_{o}(\Delta t(k+1))=\left(i_{o}(\Delta t \cdot k) / C_{o}-v_{o}(\Delta t \cdot k) / R_{o} C_{o}\right) \cdot \Delta t+v_{o}(\Delta t \cdot k)
\end{aligned}
$$

Now , the equation for the switching function $d$, is first based on an equation for the sawtooth voltage

$$
V_{\text {ramp }}(t)=V_{\text {ramp.m }} \cdot\left(t / T_{s}-\text { floor }\left(t / T_{s}\right)\right)
$$

And comparing this sawtooth voltage with the constant voltage $V$, we obtain a sequence of rectangular control pulses with switching period $T_{s}$, which reflect the functioning of the PWM system. Namely, when $\left(V-V_{\text {ramp }}(\Delta t \cdot k)\right)>0$, the function $d=" 1$ ", in the opposite case, $d=" 0$ ", that is, it would be written in the Matlab as:

$$
d(\Delta t \cdot k)=\left(\left(V-V_{\text {ramp }}(\Delta t \cdot k)\right)>0\right)
$$

It is obvious, that by changing the value of $V$, the magnitude of the duty cycle $D(d=" 1$ ") of the control system can be altered. We will complement (2) and (4) by an equation of a closed system of control with a proportional regulator, and with a dimensionless gain factor of $1 / T$ :

$$
v(\Delta t \cdot k)=\left(V_{r e f}-K_{1} \cdot v_{o}(\Delta t \cdot k)\right) \cdot \frac{1}{T}
$$

Proceeding to the form of writing of the variables in Matlab and rewriting the set of equations with a closed control system takes on the form at the next, $k+1$, step is:

$$
\begin{aligned}
& i_{o}(k+1)=\left(d(k) \cdot V_{i n} / L_{o}-v_{o}(k) / L_{o}\right) \cdot \Delta t+i_{o}(k) ; \\
& v_{o}(k+1)=\left(i_{o}(k) / C_{o}-v_{o}(k) / R_{o} C_{o}\right) \cdot \Delta t+v_{o}(k) ; \\
& v(k+1)=\left(V_{r e f}-K_{1} \cdot v_{o}(k)\right) / T ; \\
& d(k+1)=\left(\left(v(k)-V_{r a m p}(k)\right)>0\right) ;
\end{aligned}
$$

Introduce the base values of voltage, current, and time: $V_{B}=V_{i n}, I_{B}=V_{i n} / R_{o}, T_{B}=T_{s}$ respectively. With this, in relative units, equation (6), which is suitable for Matlab calculations, assumes the following form:

$$
\begin{aligned}
& x 1(1)=0 ; x 2(1)=0 ; x 3(1)=0 ; d(1)=1 ; \\
& \text { for } \quad k=1: 8 \cdot 10^{5} ; \\
& x 1(k+1)=((d(k) \cdot \tau 1-x 2(k) \cdot \tau 1) \cdot(x 1(j, k)>0)) \cdot \Delta \tau+x 1(k) \\
& x 2(k+1)=(x 1(k) \cdot \tau 2 \cdot(x 1(j, k)>0)-x 2(k) \cdot \tau 2) \cdot \Delta \tau+x 2(k) ; \\
& x 3(k+1)=\left(v_{r e f}-K_{1} \cdot x 2(k)\right) \cdot \frac{1}{T} \\
& \left.d(k+1)=\left((x 3 k)-0.05 \cdot v_{\text {ramp }}(k)\right)>0\right) \\
& \text { end }
\end{aligned}
$$

where $x 1(k)=i_{o}(k) / I_{B}, \quad x 2(k)=v_{o}(k) / V_{B}, \quad x 3(k)=v(k) / V_{B}, \quad \tau 1=R_{o} \cdot T_{s} / L_{o}, \quad \tau 2=T_{s} / R_{o} C_{o}, \quad v_{r e f}=V_{\text {ref }} / V_{B}$, $v_{\text {ramp }}(k)=V_{\text {ramp }}(k) / V_{B}, \quad u_{\text {in }}=v_{\text {in }} / V_{B}, \Delta \tau=0.5 \cdot 10^{-3}, K_{1}=0.5, \quad T=0.1$. 
The factor $(x 1(j, k)>0)$ corresponds to the fact that the output current of a buck converter cannot be negative.

Being the basis of a mathematical model of the buck converter, equation (7) make it possible to calculate all the voltages and currents on the elements of the diagram in Fig. 1. Thus, for example, Fig. 2 (a) and (b) show the results of the Matlab calculations of the relative value of the current $x 1(k)\left(i_{o}\right)$ and the relative value of the voltage $x 2(k)$ for two values of the relative input voltage $u_{i n}: u_{i n}=1.5$ and $u_{i n}=2.0$. Here the values of the constants $\tau 1=4, \tau 2=0.025$, as well as $V_{B}=20 \mathrm{~V}, I_{B}=2 \mathrm{~A}, T_{S}=20 \cdot 10^{-6} \mathrm{~s}, V_{\text {ramp.m }}=10 \mathrm{~V}$.

Fig. 2 (a) and (b) shows that for the first value of the relative input voltage, $u_{i n}=1.5$, the pulses of the output current change with the periodicity $T_{S}$ of the as the switching pulses, and the lower value of the current pulsation is always the same, $i_{o}\left(n T_{s}\right)=i_{o}\left((n+1) T_{s}\right)$, where $n$ is an integer (see Fig. 2(a)). On the other hand, for the second value of the relative input voltage, $u_{i n}=2.0$, the output current pulses change with a doubled periodicity, $2 T_{S}$ relative to the switching pulses, and the lower value of the current pulsation at these moments has two different values, $i_{o}\left(n T_{s}\right)$ and $i_{o}\left((n+1) T_{s}\right)$ (see Fig. 2(b)), and the first bifurcation and the doubling of the period occurs. Fig. 2, c,d shows the same processes given also in relative units, which have been obtained using the Matlab-Simulink program, and which coincide totally with the results of Fig. 2, a, b.
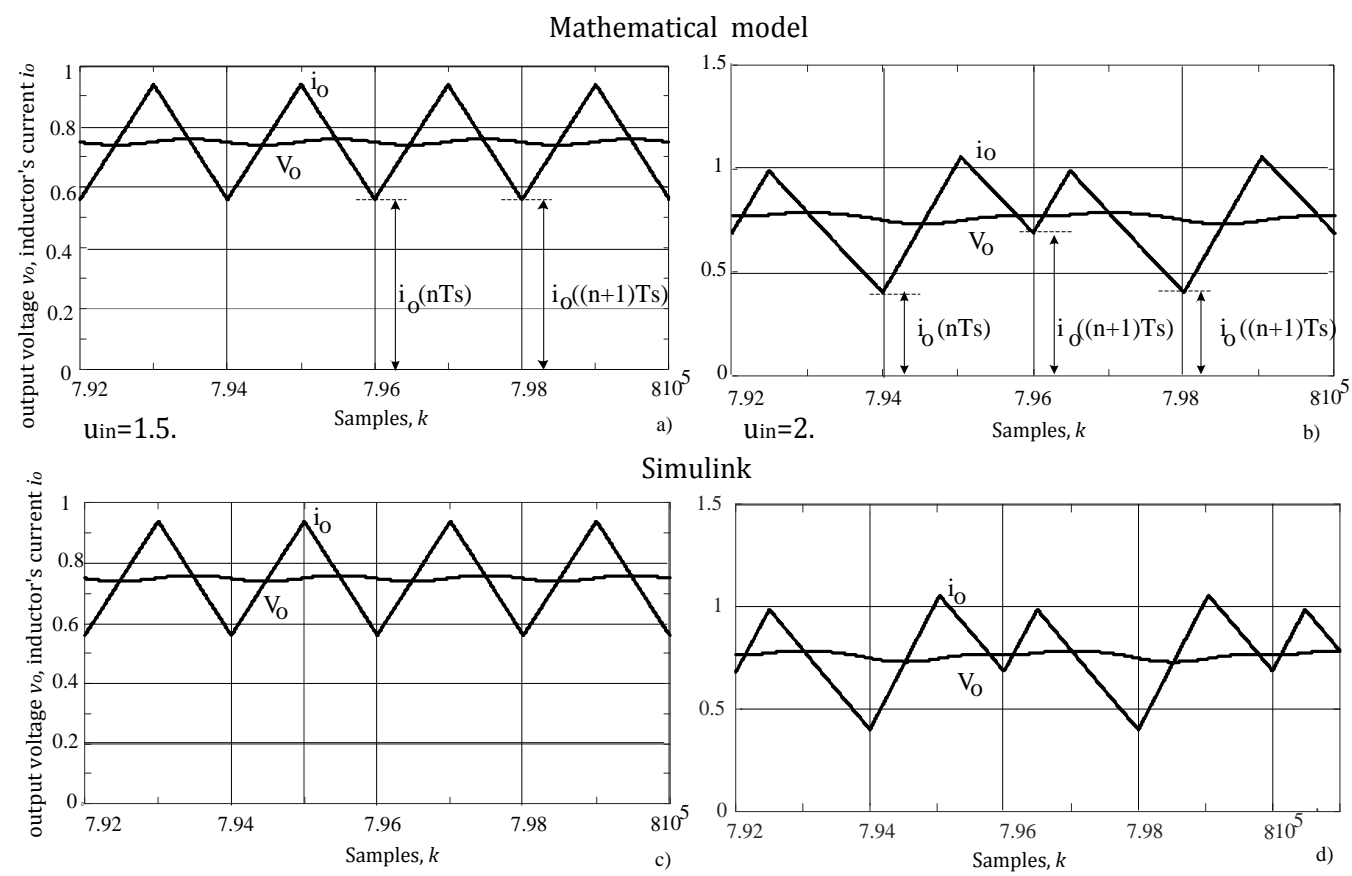

Fig. 2. (a) relative output voltage and current when $u_{\text {in }}=1.5$; (b) relative output voltage and current when $u_{\text {in }}=2 ;(\mathrm{c})$, (d) the same processes in Matlab-Simulink program.

In order to trace the whole process of changes of the output current upon changes of the input voltage in a wide range, we will calculate (7) both upon changes of the parameter $k$ (time), and upon changes of the value of the input voltage for each time cycle. The system of equations for simultaneous calculations of both cycles assumes the form: 


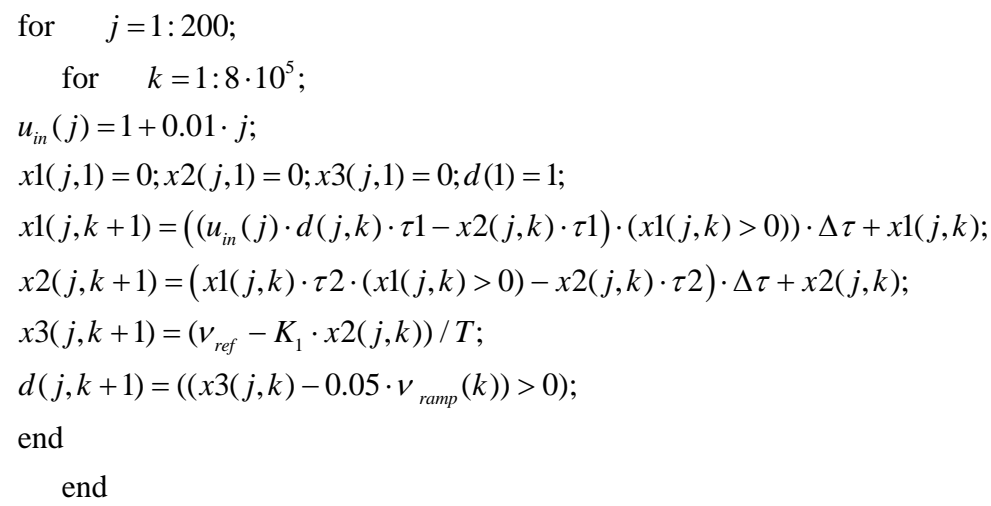

Fig. 3 shows the results of these calculations, obtained with a simple program:

$$
\begin{aligned}
& j=1: 100 ; \\
& m=8 \cdot 10^{5}-[1: 50] \cdot 2000 ; \\
& w 1=x 1(j, m) ; \\
& \operatorname{plot}\left(j, w 1(:,:),{ }^{\prime} *^{\prime}\right) ;
\end{aligned}
$$

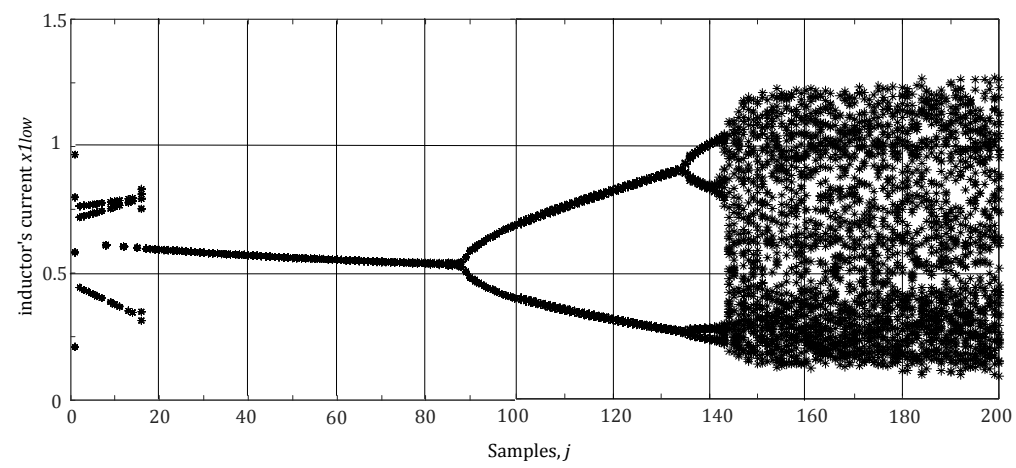

Fig. 3. Bifurcation diagram of the inductor current $i_{o}$ with input voltage variation.

First of all, let us look at the segment of the graph when $j$ (the input voltage $u_{i n}(j)=1+0.01 \cdot j$ ) changes from 20 to 88; here we see that the relative current $x 1\left(i_{o}\right)$ changes periodically, with the period equaling the period of switching, $T_{\mathrm{S}}$, that is, the lower values $x 1_{\text {low }}$ of the current pulses are the same. Further, for $j>88$ the period of repetition of the lower values of pulses doubles, then we clearly see the quadrupling of the period $(j>135)$, the beginning of its octupling and the transition to a chaotic mode. The general pattern is somewhat distorted for calculation points with $j<20$, where the regular mode is alternating with single points of the period's multiplication, which, in our opinion is due to some accidental malfunctions in the process of calculations. Note that an increasing of the value of $T$ results in an increasing of the values of $\mathrm{j}$, for which the bifurcation mode begins, that is, in the shift of the graph to the right, and the diminishing $T$ leads to a reversal in the process.

\section{A Method for Removing Bifurcations and Chaotic Modes for a P-Regulator in a Buck Converter with the Closed Loop Feedback}

In order to remove the bifurcations and chaotic modes in the above-mentioned buck converter, let us first change the diagram of a buck converter with the closed loop feedback to the one as in Fig. 4(a).

As shown in Fig. 4, the new modified circuit is supplemented by yet another switch, So, which closes periodically for a short interval of time (approx. $t \approx(0.1-0.3) T_{S}$ ) immediately before the next switching pulse of the main switch $S$. An additional diode Do prevents the discharge of the capacitor Co to the closed switch 
So. Since, in the continuous mode, during the time interval in which the diode $D$, acts as a conductor, at this time the inductor Lo turns to be short-circuited, and in the ideal case, the current through it does not change. As is seen in Fig. 4(a), in order to form the aforesaid pulse, the same sawtooth voltage $V_{\text {ramp }}$ in the other block $P W M o$ is being compared with the voltage $V_{\text {refo, }}$, which is somewhat less in value than the amplitude of $V_{\text {ramp }}$, so in accordance with the polarity shown in Fig. 4(a), the short pulse of switch So immediately before the main pulse on the switch $S$. It is easy to see that when we denote the value of the duty cycle of the pulse at switch So through Do, the formula for the output voltage is of the form:

$$
V_{o}=V_{i n} D /\left(1-D_{o}\right)
$$

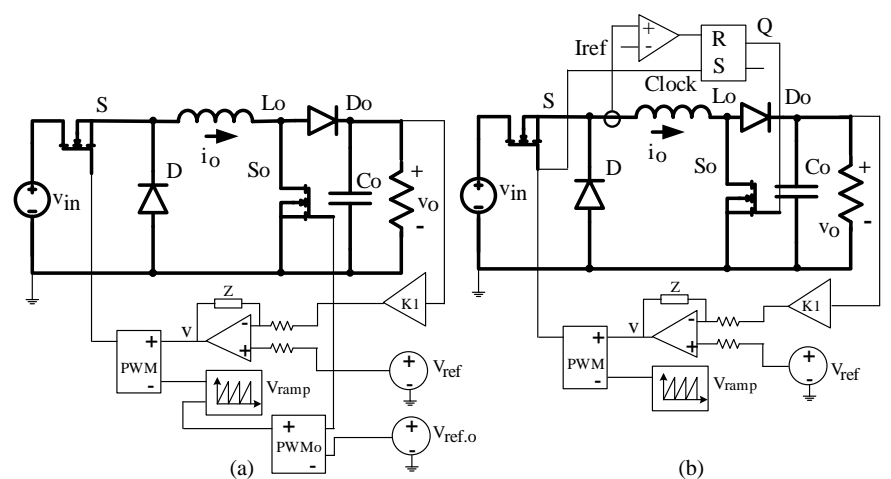

Fig. 4. (a) A modified buck converter for P-regulator (b) A modified buck converter for I-regulator.

The system of equations for the model of the new system is given in (11). It differs from the model (9) only by the setting of the value $u_{\text {ref.o }}(j)=0.45-0.0005 \cdot j$, and by the factor $\left(u_{\text {ref.o }}(j)>0.05 \cdot v_{\text {ramp }}(k)\right)$ in the first and the second equations, which "switches off" the resistance Ro and the capacitor Co from the rest of the circuit for the time of the pulse on So.

The results of the calculations in the form of a plot of the dependence of the lower values of the pulses of the current $x 1\left(i_{0}\right)$ in the inductor on the magnitude of the input voltage determined by the parameter $j$, are given in Fig. 5. As compared with Fig. 3, in the latter case bifurcations, and, all the more so, chaotic modes all over the range of the changes of the supply voltage (in relative units from 1 to 3 ) are absent. The calculations were carried out in two stages: first upon the changing of the input voltage from 1 to 2 , and then, from 2 to 3 . In the first case, the reference voltage for the forming of the pulse of the auxiliary switch So $u_{\text {ref.o }}(j)=0.45-0.0005 \cdot j$, and in the second, $u_{\text {ref.o }}(j)=0.40-0.0005 \cdot j$. The results of the calculations were then joined in a single plot in Fig. 5 .

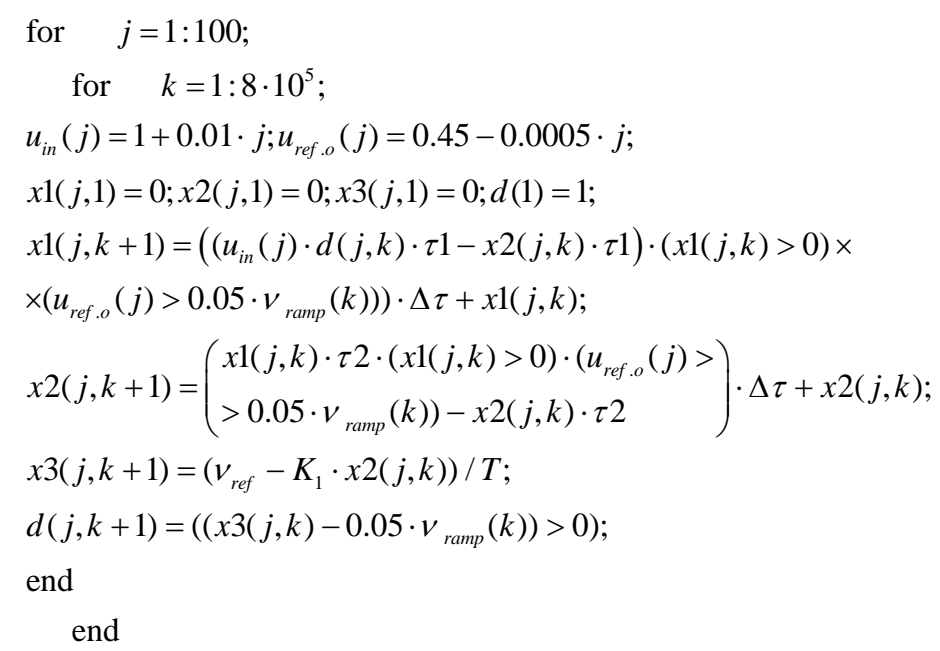




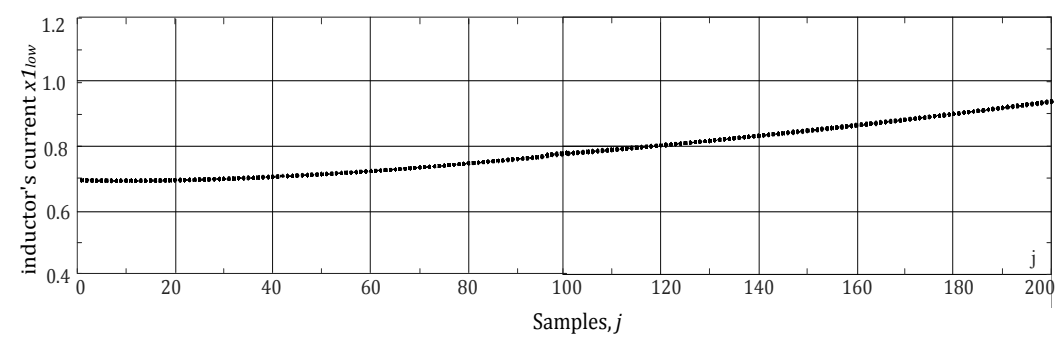

Fig. 5. The lower values of the inductor current after the elimination of bifurcations.

\section{The Description of a Mathematical Model of the Buck Converter with the Closed Loop Feedback and an Integral (Proportional-Integral) Regulator (I-Regulator, PI-Regulator)}

Consider now the same mode of the changing of the input voltage, with an I-regulator in the closed loop feedback system (see Fig. 1), the feedback of the amplifier $Z$ consists of the capacitor $C$, so the gain factor of the amplifier $K=1 / R C=1 / T_{r}$ (and the time constant equals $T_{r}=R C$, or, in relative units, $T=R C / T_{S}$ ).

In this case, the system of equations describing the functioning of the model is similar to (8), only the third equation, which describes the output of the integral regulator, $x 3(j, k)$ will be different:

$$
x 3(j, k+1)=\left(\left(v_{r e f}-K_{1} \cdot x 2(j, k)\right) \cdot \frac{1}{T}\right) \cdot \Delta \tau+x 3(j, k) ;
$$

This system of equations makes it possible to determine the output voltage and current for any value of the input voltage within the range from 1 to 3 , and for any value of the time constant of the amplifier. The calculations show that for the time constant of the amplifier, $T_{r}=R C \geq 2 \cdot 10^{-4}$, the system is stable and in a steady-state a periodic mode of the output current and voltage establishes itself, with the frequency of pulses that equals the frequency of switching (Fig. 6(a), shows the values of the output current and voltage at $u_{i n}=3$ ). As $T$ decreases, the mode becomes non-stable, and undamped, then low-frequency auto-oscillation establish themselves (Fig. 6( b) shows the results for, $u_{i n}=3, T_{r}=1 \cdot 10^{-4} \mathrm{~s}$ ). Fig. 6 (c) and (d) shows the same processes, obtained by Matlab-Simulink program, which are shown to be identical to the results in Fig. 6 (a) and (b).
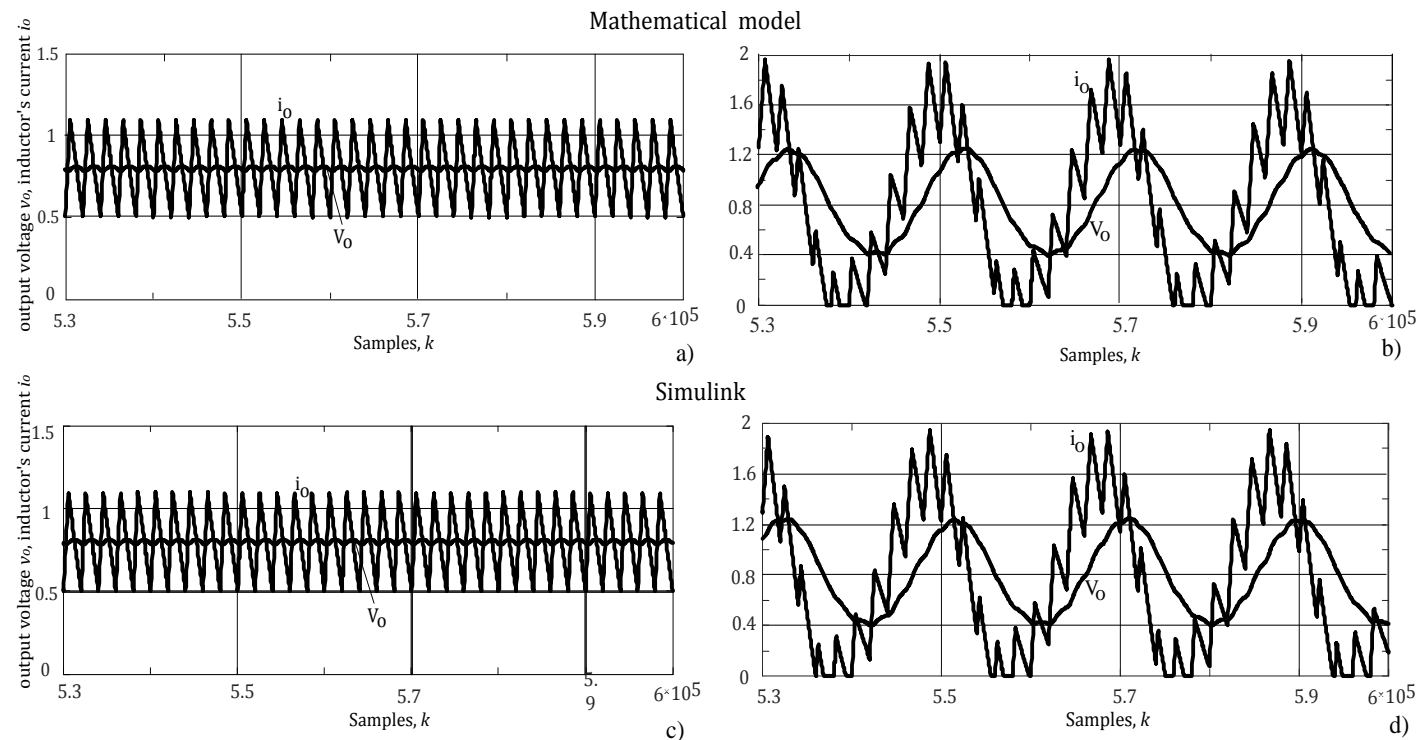

Fig. 6. (a) relative output voltage and current when $u_{i n}=3, T_{r}=R C=2 \cdot 10^{-4} \mathrm{~s}$; (b) relative output voltage and current when $u_{i n}=3, T_{r}=1 \cdot 10^{-4} s$; (c), (d) the same processes in Matlab-Simulink program. 
The frequency of auto-oscillations does not depend on the magnitude of the input voltage, despite the fact that their amplitude increases with an increase of the value $u$. It is seen from Fig. 7, which shows the trajectory of the changes in the values of the output current at the moments of the entering of switching pulses.

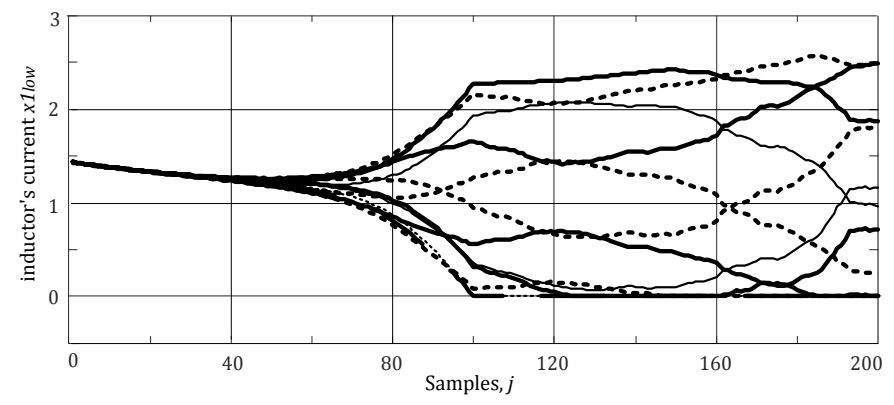

Fig. 7. Bifurcation diagram of the inductor current $i_{o}$ with input voltage variation for I-regulator an

$$
T_{r}=1 \cdot 10^{-4} \mathrm{~s} .
$$

The values are conditionally symmetric with respect to the mean value of the output current are marked by the same line style. The number of branches in the plot after its branching at $j \approx 40, u=1.4$, remains unchanged, and the values of some of them vanish upon an increase of $u_{i n}$. The fact that number of the plot branches remains constant indicates that the frequency of the low-frequency auto-oscillations remains constant. Note that with an integral regulator, the system does not become chaotic. The same picture is observed in the case of a PI-regulator, when the equation for the calculation of $x 3(j, k+1)$ in (8) assumes the form (here $1 / T_{p}$ is the gain factor of the P-regulator):

$$
x 3(j, k+1)=\left(\left(v_{r e f}-K_{1} \cdot x 2(j, k)\right) \cdot \frac{1}{T}\right) \cdot \Delta \tau+x 3 \cdot(j, k)\left(1+\Delta \tau \cdot \frac{1}{T_{p}}\right) ;
$$

\section{A Method to Eliminate Instabilities of an I-Regulator in Buck Converters with Closed Loop Feedback}

In the case of a system with an I-regulator, the current of the inductor has a deeply oscillating character (as seen Fig. 6(b)), therefore, instead of supplying an additional pulse according to the diagram in Fig. 4(a) at the fixed moments of time, it should be supplied at the moments when the lower level of current in the inductor reaches a certain value, as is shown in the diagram of Fig. 4(b). In order to do so, the control system is supplemented by a comparator whose input receives the inductor's current $x 1,\left(i_{o}\right)$ and the value of the limiting current, $\boldsymbol{l}_{\text {ref.o }}$, as well as by a RS-trigger that forms the necessary pulse at the switch So. The back return of the trigger is made by the Clock pulses that are formed after the back front of the sawtooth voltage. This makes it possible to eliminate low-frequency oscillations of the output current over all the range of the input voltage, as is shown clearly from calculations even upon a considerable decrease of the time constant of the integrator (see Fig. 8). Fig. 9 shows the lower values of the pulsation of the output current over all the range of voltage changes upon changing the limiting current according to the formula $\boldsymbol{l}_{\text {ref.o }}(j)=0.75-0.00005 \cdot j$, that is with a certain decrease of the current, as the input voltage increases. The plot shows the fact that all over the range of the changes of the input voltage the current is in a steady-state and the frequency of pulses equals the frequency of switching.

The system of equations (11) changes in respect to the setting of the limiting current and assumes the following form: 


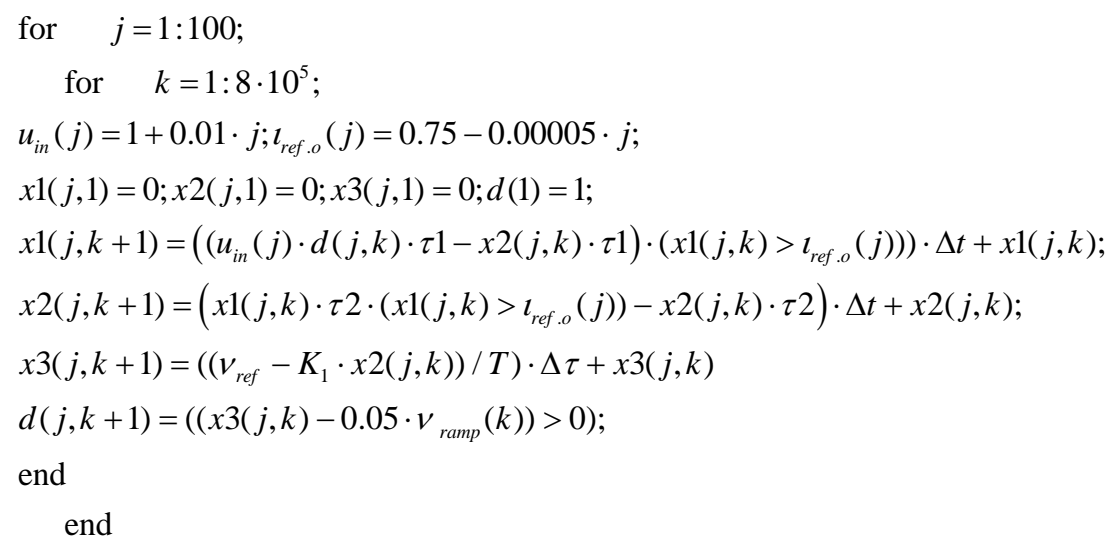

Thus, the proposed methods of influencing the inductor's current using an additional switch eliminate completely both the chaotic mode and undamped low-frequency oscillations in a wide range of the variation of the input voltage and the regulators' parameters.

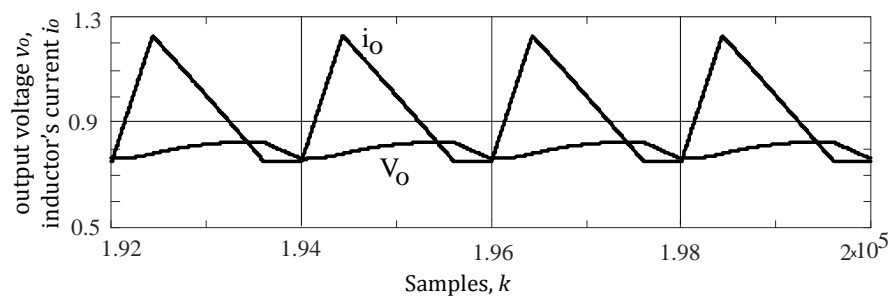

Fig. 8. Relative output voltage and current when $u_{\text {in }}=3, i_{r e f .0}=0.75, T_{r}=R C=5 \cdot 10^{-5} \mathrm{~s}$ in modified buck converter for I-regulator.

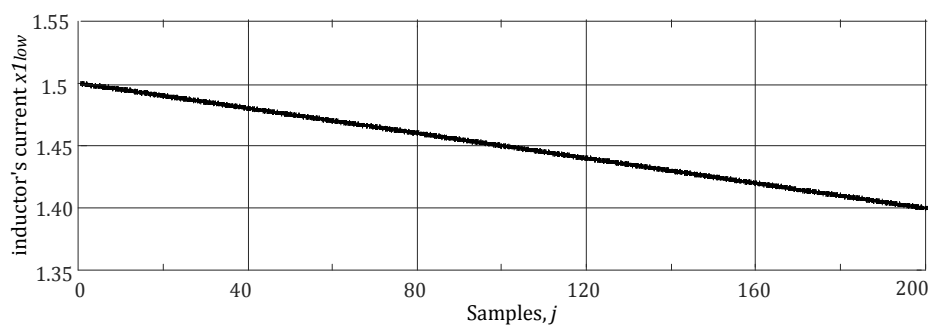

Fig. 9. The lower values of the inductor current after the elimination of bifurcations for I-regulator.

\section{Buck Converter with a Closed Loop Control System Combined with Parametric Control}

Since in the static mode $V_{o}=D \cdot V_{i n}$, and, on the other hand, at the linear sawtooth voltage in a PWM system $D=V_{\text {contr }} / V_{\text {ramp.m. }}$, then maintaining $V_{\text {ramp.m }}=V_{\text {in }}$ for any input voltage, we obtain $V_{o}=V_{\text {contr }}$, namely, the output voltage in an ideal case would be maintained constant and equal to the control voltage at any values of the input voltage. The above-mentioned does not take into account changes in the load, and internal losses of voltage, therefore, to compensate these changes, we will preserve the above considered closed systems of control combined with parametrical control.

If the sawtooth voltage is given by the formula (3) with $V_{\text {ramp.m }}=1 \mathrm{~V}$, then in the system of equations for P-regulator and I-regulator (8) and (12) only the last equation would be changed, assuming the form:

$$
d(j, k+1)=\left(\left(\left(x 3(j, k)+v_{r e f}\right)-u_{i n}(j) \cdot v_{\text {ramp }}(k)\right)>0\right) ;
$$


The calculations show that for such a control in all the range of variations of the input voltage, a usual mode of the pulsation of the inductor's current is maintained, with the switching frequency (see Fig. 10, analogous to Fig. 5 and Fig. 9). It can be easily explained, on the one hand, the output signals of P-regulator and I-regulator comprise a small part of the general control signal, and, on the other hand, the sawtooth voltage itself increases, and there are no conditions for the multiplication of the period of pulses.
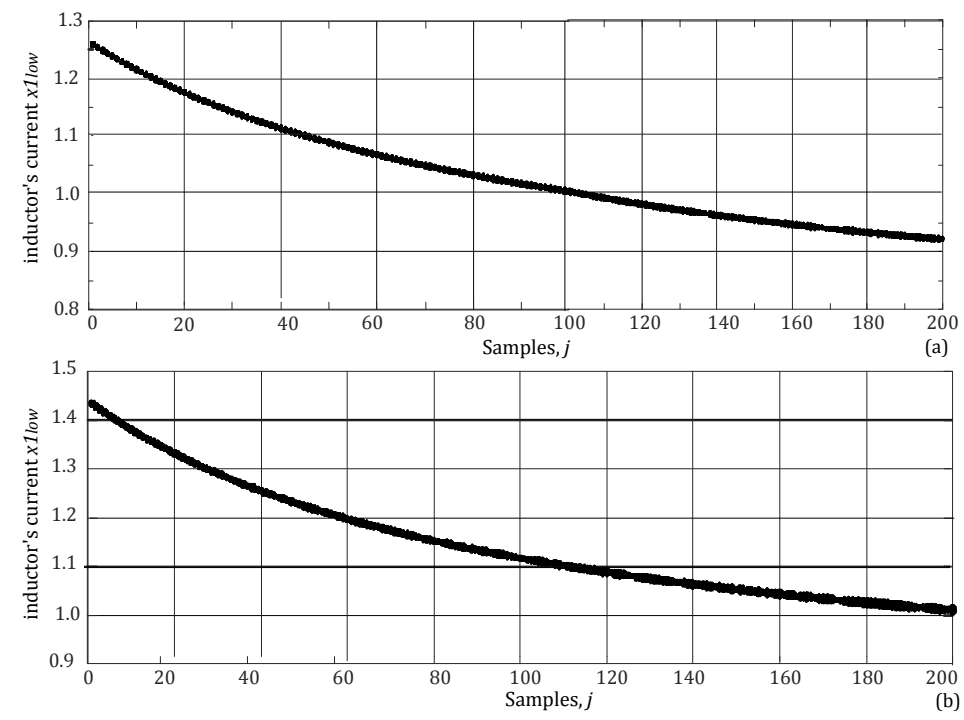

Fig. 10. The lower values of the inductor current after the elimination of bifurcations with parametric control, (a) for P-regulator, (b) for I-regulator.

\section{Conclusions}

1. A simple mathematical model of a buck converter with feedback has been developed on the basis of the Matlab platform, which makes it possible to obtain not only all the modes of the converter functioning, including the chaotic or undamped oscillatory ones, but also directly using this model, to carry out all the necessary numerical and graphical processing of the results.

2. Two kinds of regulators in the feedback circuit - the proportional, and the integral (or proportional-integral) ones have been considered and analyzed, for which two types of differing in principle processes have been obtained: in the first case bifurcations and the onset of chaos upon the growth of the supply voltage, and undamped oscillations of the reactor current, in the second case.

3. For each of the types of control considered, there have been proposed the method either of the synchronization of the chaotic mode, or the complete elimination of oscillations; both have shown their efficiency. In both cases, the methods are based on the effect on the ripple of the reactor current and, accordingly, on the reactive power of the current ripple circulating between the source and the converter.

\section{References}

[1] Gleick, J. (1993). Chaos: Making a New Science (p. 352). London: Abacus.

[2] Haken, H. (1983). Synergetics: An Introduction (p. 390). Berlin: Springer.

[3] Prigogine, I. (1997). The End of Certainty: Time, Chaos and the New Laws of Nature (p. 207). New York.

[4] Prigogine, I., \& Stengers, I. (1984). Order out of Chaos (p. 349). London: Heinemann.

[5] Brillouin, L. (1963). Science and Information Theory (2nd ed.). Chicago: IL Academic Press.

[6] Doyle, B. (2011). The Scandal in Philosophy. Cambridge: MA, USA, I-Phi Press. 
[7] Deane, J., \& Hamill, D. (1990). Instability, subharmonics and chaos in power electronic systems. IEEE Transactions on Power Electronics, 5(3), 260-267.

[8] Deane, J. H. B. (1992). Chaos in current-mode controlled boost DC-DC converter. IEEE Transactions on Circuits and Systems-1, 3(8), 680-683.

[9] Hamill, D., Deane, J., \& Jefferies, D. (1992). Modeling of chaotic DC-DC converters. IEEE Transactions on Power Electronics, 7(1), 25-36.

[10] Tse, C. K. (2003). Chaos in power electronics. Proceedings of the IEEE ISCAS, Tutorial Notes (p. 28).

[11] Tse, C. K. (2004). Complex Behavior of Switching Power Converters (p. 266). CRC Press.

[12] Iu, H. H. C., \& Tse, C. K. (2000). A study of synchronization in chaotic autonomous C'uk DC/DC converters. IEEE Transactions on Circuits and Systems-I: Fundamental Theory and Applications, 47(6), 913-918.

[13] Olivar, G. (1997). Chaos in the buck converter. Universitat Politechnika de Catalunya.

[14] Ott, E., Grebogi, C., \& Yorke, J. (1990). Controlling chaos. Physics Review Letters, 64(11), 1196-1199.

[15] Yuan, G., Banerjee, S., Ott, E., \& Yorke, J. A. (1998). Border-collision bifurcations in the buck converter. IEEE Transactions on Circuits and Systems -I: Fundamental Theory and Applications, 45(7), 707-716.

[16] Pyragas, K. (1992). Continuous control of chaos by self-controlling feedback. Physics Letters A, 170, 421-428.

[17] Kocewiak, L. (2008). Bifurcations and chaos in a DC-DC buck converter. Elektronika, 10, 105-110.

[18] Chang, C.-Y., Zhao, X., Yang, F., \& Wu, C.-E. (2016). Bifurcation and chaos in high-frequency peak current mode Buck converter. Chinese Physics B, 25(7).

[19] Morcillo, J. D., Olivar, G., \& Angulo, F. (2014). Feed-forward chaos control of a buck converter. International Symposium on Nonlinear Theory and Its Applications (NOLTA 2014). Luzern, Switzerland.

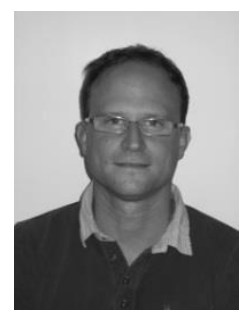

Yuval Beck was born in Tel Aviv, Israel, on November 30, 1969. He received the B.Sc degree in electronics and electrical engineering from Tel Aviv University in 1996; the M.Sc. degree in 2001, and the Ph.D. degree on the subject of ground currents due to lightning strokes in 2007 from Tel Aviv University as well. Since 1998, he has been with the Interdisciplinary Department, the Faculty of Engineering, Tel Aviv University. In 2008 joined HIT-Holon Institute of Technology, Holon, Israel, as a lecturer and from 2010 was acting as the head of Energy and Power Systems Department at the Faculty of Engineering.

Since 2017 he joined the Physical Electronics Department at the School of Elevtrical Engineering at Tel Aviv University. His research interests include smart grid technologies, lightning discharge phenomena, lightning protection systems, power electronics, and photovoltaic systems.

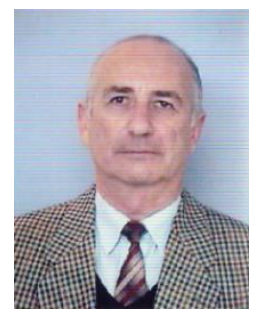

Yefim Berkovich was born in the Ukraine in 1937. He received the electrical engineer diploma from the Lvov Polytechnic Institute in 1959 and the degrees of candidate of technical sciences from the All-Union Electrotechnical Institute, Moscow, in 1975, and doctor of technical sciences from the Institute Electrodynamics, Kiev, in 1990.

During the period 1959-1966 he worked at the Special Design Bureau of Power Electronics, in Mordovia, Russia, and during the period 1966-1996, in Electrotechnical Works of Power Electronics, in Tallinn, Estonia (from 1995 also as professor at Tallinn Polytechnic University). Since 1997 he has been with the Academic Institute of Technology, Holon, Israel. His research interests include power electronics systems such a dc-dc, ac-dc, and ac-ac converters. Dr. Berkovich has published over 100 papers in electrical engineering. 